\title{
Noise switching at a dynamical critical point in a cavity-conductor hybrid
}

\author{
Andrew D. Armour, ${ }^{1}$ Björn Kubala, ${ }^{2}$ and Joachim Ankerhold ${ }^{2}$ \\ ${ }^{1}$ School of Physics and Astronomy and Centre for the Mathematics \\ and Theoretical Physics of Quantum Non-Equilibrium Systems, \\ University of Nottingham, Nottingham NG7 2RD, UK \\ ${ }^{2}$ Institute for Complex Quantum Systems and IQST, \\ Ulm University, Albert Einstein-Allee 11, 89069 Ulm, Germany
}

(Dated: December 20, 2017)

\begin{abstract}
Coupling a mesoscopic conductor to a microwave cavity can lead to fascinating feedback effects which generate strong correlations between the dynamics of photons and charges. We explore the connection between cavity dynamics and charge transport in a model system consisting of a voltagebiased Josephson junction embedded in a high-Q cavity, focussing on the behavior as the system is tuned through a dynamical critical point. On one side of the critical point the noise is strongly suppressed, signalling the existence of a novel regime of highly coherent transport, but on the other side it switches abruptly to a much larger value. Using a semiclassical approach we show that this behavior arises because of the strongly nonlinear cavity drive generated by the Cooper pairs. We also uncover an equivalence between charge and photonic current noise in the system which opens up a route to detecting the critical behavior through straightforward microwave measurements.
\end{abstract}

\section{INTRODUCTION}

Combining voltage-biased mesoscopic conductors with high-Q superconducting cavities leads to a unique class of hybrid devices. Recent experiments on such systems, using conductors such as Josephson junction (JJ) devices [1-3], or quantum dots $[4,5]$, have demonstrated that coherent interactions between a conductor and a cavity can generate large non-equilibrium photon populations. Parallels can be drawn between cavity-conductor hybrids and quantum optical devices such as the micromaser in which a stream of excited atoms flows through an optical cavity $[6,7]$; in both cases the photons in the cavity act back on the photon generation process leading to strongly nonlinear dynamics and dissipative phase transitions. However, whilst the center of mass motion of atoms flowing through a micromaser is independent of the interaction with the cavity, the charge current in cavity-conductor systems can be strongly correlated with the cavity state leading to a rich interplay between the quantum transport of charges and the quantum optics of photons [8].

The interaction between the charge current and the cavity dynamics is particularly interesting in the case of a voltage biased JJ in series with a microwave cavity [2, 3, 9-12]. The charge-photon interactions can be tuned using the voltage to generate an effective cavity drive which is strongly nonlinear [13-15], leading to dissipative dynamics and critical behavior very different from commonly studied nonlinear quantum oscillator systems [16-22]. Furthermore, all of the energy from the voltage source can be converted into photons leading to an average dc current that is strictly proportional to the rate at which photons are produced $[2,11]$, unlike other conductors such as semiconductor quantum dots, where typically only a small fraction of the charges generate photons [4, 23]. Theoretical [13, 14, 24-31] and experimental studies $[2,3,11,32]$ of JJ-cavity systems over the last few years have investigated the complex nonlinear quantum dynamics that arises and the non-classicality of the microwaves produced.

In this paper we explore the interplay between the charge and photon dynamics in voltage-biased JJ-cavity systems. The nonlinear cavity drive generated by the Cooper pairs leads to a transition from incoherent single Cooper pair transfer [11] to a novel regime of strongly coherent transport [33], signalled by suppression of current fluctuations. However, when the system reaches a dissipative critical point the character of the transport changes and the current noise can switch abruptly from strongly sub-Poissonian to strongly super-Poissonian. The current noise suppression on one side of the critical point relies on a decoupling of the current fluctuations from the critical slowing down, something which is not seen at dissipative transitions in other systems where a mesoscopic conductor generates a non-equilibrium photon or phonon population [34-41]. We use a semiclassical approach to understand the key elements of the behavior of the system and to show how they are linked to the particular form of the nonlinearity generated by the interactions between the photons and Cooper pairs. We also demonstrate that the flow of photons through the cavity becomes closely connected with the charge dynamics, providing a convenient way of detecting the coherent transport regime.

\section{JOSEPHSON-CAVITY SYSTEM}

The model system we study consists of a JJ in series with an $L C$ oscillator to which a (sub-gap) voltage bias, $V$, is applied $[2,11]$. The oscillator is assumed to be one of the modes of a high-Q superconducting microwave cavity which is weakly coupled to a transmission line through which photons leak out of the system [13] at a rate $\gamma$. The Hamiltonian of this JJ-oscillator 
system contains a time-dependent effective drive term $H=\hbar \omega_{0} a^{\dagger} a-E_{J} \cos \left(\omega_{J} t+\varphi\right)[13,14]$, where $a$ is the lowering operator for the oscillator which has frequency $\omega_{0}=1 / \sqrt{L C}, E_{J}$ is the Josephson energy of the junction and $\omega_{J}=2 \mathrm{eV} / \hbar$ the Josephson frequency set by the bias voltage. The drive term is highly nonlinear because the phase of the JJ is locked to the phase of the oscillator [42] $\varphi=\Delta_{0}\left(a^{\dagger}+a\right)$ with $\Delta_{0}=\left(2 e^{2} / \hbar\right)^{1 / 2}(L / C)^{1 / 4}$ the oscillator's zero point flux fluctuations in units of the flux quantum.

Moving to a frame rotating at $\omega_{J}$ and making a rotating wave approximation leads to an approximate Hamiltonian for the region close to the one-photon resonance $[13,14]$ where $\omega_{J} \simeq \omega_{0}$,

$$
H_{\mathrm{RWA}}=\hbar \delta a^{\dagger} a+i \frac{\tilde{E}_{J}}{2}:\left[a^{\dagger}-a\right] \frac{J_{1}\left(2 \Delta_{0} \sqrt{a^{\dagger} a}\right)}{\left(a^{\dagger} a\right)^{1 / 2}}:,
$$

here the renormalized Josephson energy is defined as $\tilde{E}_{J}=E_{J} \mathrm{e}^{-\Delta_{0}^{2} / 2}$, the detuning is given by $\delta=\omega_{0}-\omega_{J}$ and colons imply normal ordering. Including weak coupling between the oscillator and its surroundings (i.e. the transmission line), assumed to be at zero temperature for simplicity, leads to a master equation for the cavity,

$$
\dot{\rho}=-\frac{i}{\hbar}\left[H_{\mathrm{RWA}}, \rho\right]+\frac{\gamma}{2}\left(2 a \rho a^{\dagger}-a^{\dagger} a \rho-\rho a^{\dagger} a\right) .
$$

By considering the phase difference across the JJ we can write down the charge current operator, $I_{J}=$ $(2 e / \hbar) E_{J} \sin \left[\omega_{J} t+\Delta_{0}\left(a+a^{\dagger}\right)\right]$. Close to the onephoton resonance the operator for the dc current of Cooper pairs is obtained by moving to the rotating frame and retaining the constant part:

$$
I_{C P}=\frac{e \tilde{E}_{J}}{\hbar}: \frac{J_{1}\left(2 \Delta_{0} \sqrt{a^{\dagger} a}\right)}{\left(a^{\dagger} a\right)^{1 / 2}}\left[a+a^{\dagger}\right]: .
$$

The current operator and the cavity occupation number, $n=a^{\dagger} a$, are connected by the relation $I_{C P} / 2 e=$ $i\left[H_{\mathrm{RWA}}, n\right] / \hbar$, reflecting the fact that each Cooper pair in the dc current generates one photon. Hence, in the steady state the average cavity occupation number and current are strictly proportional, $\gamma\langle n\rangle=\left\langle I_{C P}\right\rangle / 2 e$.

\section{CHARGE TRANSPORT AT THE DISSIPATIVE CRITICAL POINT.}

Increasing $E_{J}$ enhances the tunneling of Cooper pairs, and we naturally expect this to lead to a growth of the current, hand in hand with the photon population of the cavity. However, this intuitive picture breaks down when the strong nonlinearities in the system start to play a role. When the system is tuned to resonance, $\delta=0$, a critical point emerges [13, 14] at $E_{J} \simeq E_{J}^{B}=$ $\hbar \gamma z_{1} \mathrm{e}^{\Delta_{0}^{2} / 2} /\left[4 J_{0}\left(z_{1}\right) \Delta_{0}^{2}\right]$ with $z_{1} \simeq 1.841$. The critical point marks a rapid saturation in the current (and photon number), shown in Fig. 1(a). The evolution of the
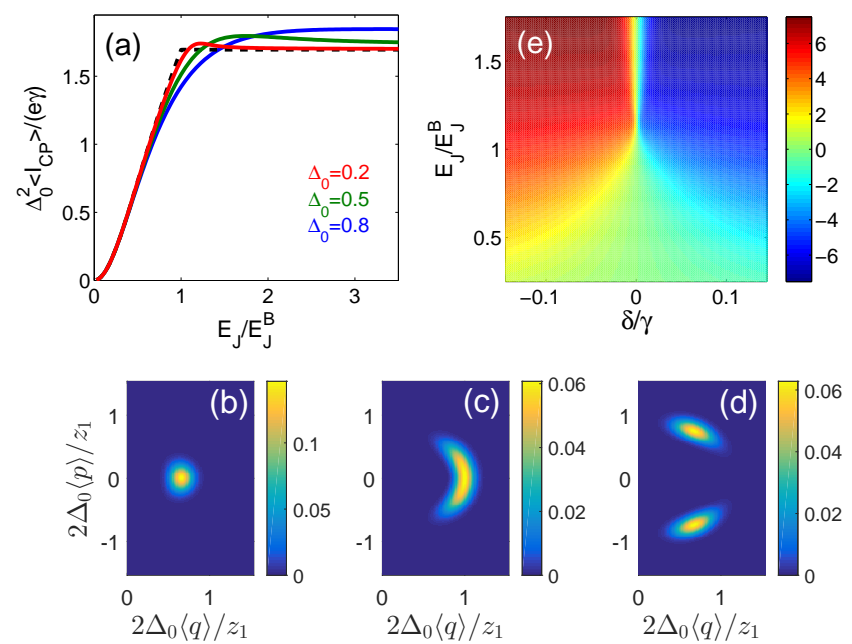

FIG. 1. (a) Average current as a function of $E_{J} / E_{J}^{B}$ for $\delta=0$; dashed lines are from the semiclassical calculation discussed below. (b), (c), (d) Corresponding evolution of the Wigner function, for $E_{J} / E_{J}^{B}=0.5, E_{J} / E_{J}^{B}=1.0, E_{J} / E_{J}^{B}=1.5$ with $\Delta_{0}=0.2$. (e) Behavior of $\langle p\rangle$ as a function of $\left(\delta, E_{J}\right)$ for $\Delta_{0}=0.1$; the system has a critical point at $\left(0, E_{J}^{B}\right)$ and a line of first order transitions along $\delta=0$ for $E_{J}>E_{J}^{B}$. Except where stated otherwise, these results are calculated numerically using the full master equation (2) [43].

Wigner function of the system with $E_{J}$, shown in Figs. 1(b)-(d), tells us that a bistability develops for $E_{J}>E_{J}^{B}$ where the steady-state becomes a mixture of two components with equal position, $\langle q\rangle=\left\langle a+a^{\dagger}\right\rangle / 2$, and opposite momenta $\langle p\rangle=\left\langle i\left(a^{\dagger}-a\right) / 2\right\rangle$.

Looking at the behavior as a function of $\delta$ as well as $E_{J}$, reveals that the critical point marks the beginning of a line of first order transitions. The bistability in the state of the system for $E_{J}>E_{J}^{B}$ and $\delta=0$ disappears rapidly with increasing $|\delta|$ : only one of the two components in the mixture survives, leading to a rapid increase (or decrease) in $\langle p\rangle$ as can be seen in Fig. 1(e). The value of $\Delta_{0}$ sets the overall size of the phase space accessible to the system. Figure 1(a) shows that the average current (and hence also the cavity occupation number) scales as $1 / \Delta_{0}^{2}$. Hence $\Delta_{0} \rightarrow 0$ acts as the 'thermodynamic' and semiclassical limit for this system [7, 21, 22].

The Cooper-pair current noise provides insight into the nature of the charge transport and into the dynamics of the system more generally [44]. It is given by

$$
S_{C P}=2 \operatorname{Re} \int_{0}^{\infty} d \tau\langle\delta I(t+\tau) \delta I(t)\rangle,
$$

where the long time $(t \rightarrow \infty)$ limit is assumed so that the system is stationary and $\delta I=I_{C P}-\left\langle I_{C P}\right\rangle$. Scaling the noise by the average current gives the Fano factor, $F_{C P}=S_{C P} /\left(2 e\left\langle I_{C P}\right\rangle\right)$. It provides a convenient way of quantifying deviations from the Poissonian limit $\left(F_{C P}=\right.$ 1 ) of uncorrelated tunneling of individual Cooper pairs, while a phase coherent supercurrent is characterized by 


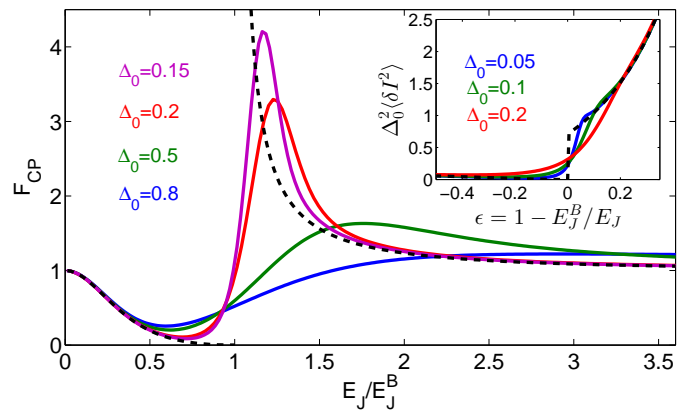

FIG. 2. Current Fano factor, $F_{C P}$ as a function of $E_{J} / E_{J}^{B}$ for a range of $\Delta_{0}$, values. The inset shows the critical scaling of $\Delta_{0}^{2}\left\langle\delta I^{2}\right\rangle$. In each case the full curves are numerical solutions of the master equation, the dashed curves are the predictions of the semiclassical calculation discussed below, and $\delta=0$.

\section{$F_{C P} \approx 0[33]$}

One intuitively expects to see a growth in the current noise as the dynamics slows down in the vicinity of dissipative transitions [34-41]. However, numerical calculations reveal a far more interesting picture. Figure 2 shows that $F_{C P} \simeq 1$ for very small, and very large values of $E_{J}$. In between, the value of $F_{C P}$ drops towards zero, implying that the flow of Cooper pairs becomes highly coherent [33], before suddenly growing for $E_{J} \simeq E_{J}^{B}$, and then decaying again. For $\Delta_{0} \ll 1$ the critical point is marked by an abrupt switching in the magnitude of the current noise.

By combining information about the magnitude of fluctuations in the current and time-scales of the system dynamics, the current noise provides a much more robust signature of the critical point than either the average current or the steady-state current fluctuations, $\left\langle\delta I^{2}\right\rangle$ (see the inset in Fig. 2).

\section{SEMICLASSICAL DESCRIPTION}

When $\Delta_{0}$ is small, as is typically the case in experiments $[2,11]$, the behavior of the system is well described using a semiclassical approach in which the classical Hamiltonian of the system, $\mathcal{H}\left(\alpha, \alpha^{*}\right)$, obtained by replacing the operator $a^{(\dagger)}$ in Eq. (1) by the complex amplitude $\alpha^{(*)}$, plays the key role (see Appendix A for details). We move to a displaced frame, $\tilde{\rho}=D^{\dagger}(\alpha) \rho D(\alpha)$, with $D(\alpha)=\mathrm{e}^{\alpha a^{\dagger}-\alpha^{*} a}$ and neglect operator terms beyond quadratic order in the Hamiltonian [18]. The value of $\alpha$ is chosen to match one of the classical fixed points of the system, solutions of $\partial_{\alpha} \mathcal{H}=-i \hbar \gamma \alpha^{*} / 2$. The semiclassical master equation is

$$
\frac{d \tilde{\rho}}{d t}=-\frac{i}{\hbar}\left[H_{2}, \tilde{\rho}\right]+\frac{\gamma}{2}\left(2 a \tilde{\rho} a^{\dagger}-a^{\dagger} a \tilde{\rho}-\tilde{\rho} a^{\dagger} a\right),
$$

with the quadratic Hamiltonian

$$
H_{2}=\left(\partial_{\alpha \alpha^{*}} \mathcal{H}\right) a^{\dagger} a+\frac{1}{2}\left[\partial_{\alpha^{*} \alpha^{*}} \mathcal{H}\left(a^{\dagger}\right)^{2}+\partial_{\alpha \alpha} \mathcal{H} a^{2}\right],
$$
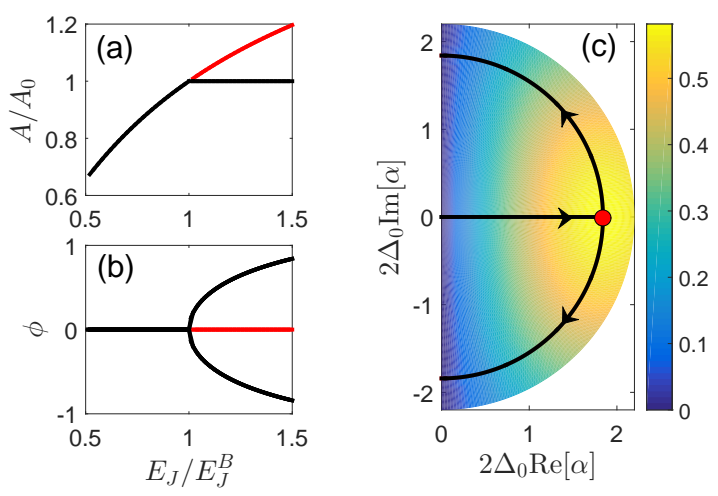

FIG. 3. Behavior of the amplitude, $A / A_{0}$, where $A_{0}=$ $z_{1} / 2 \Delta_{0}$, (a) and phase, $\phi$, (b) of the classical stable (unstable) fixed points shown as black (red) lines. (c) Classical current function, $\mathcal{I}\left(\alpha, \alpha^{*}\right) / \tilde{E}_{J}$, overlaid by the locus of fixed points (black line). The maximum in $\mathcal{I}$ coincides with the critical point (red dot). In each case $\delta=0$.

where the derivatives are evaluated at the corresponding fixed point [cf. Eqs. (A3) and (A4)]. The eigenvalues,

$$
-\Gamma_{ \pm}=-\frac{\gamma}{2} \pm \frac{1}{\hbar} \sqrt{\left|\partial_{\alpha \alpha} \mathcal{H}\right|^{2}-\left(\partial_{\alpha \alpha^{*}} \mathcal{H}\right)^{2}} .
$$

determine the stability of the fixed points and the dynamics of correlation functions.

On-resonance, one has $\mathcal{H}=\tilde{E}_{J} \sin \phi J_{1}\left(2 \Delta_{0} A\right)$, where the (real) amplitude $A$ and phase $\phi$ are defined via $\alpha=$ $A \mathrm{e}^{i \phi}$. Since fixed points obey the condition $[45] \partial_{A} \mathcal{H}=$ 0 , they occur, due to the anti-symmetry $\mathcal{H}(A,-\phi)=$ $-\mathcal{H}(A, \phi)$, either with zero phase or in pairs with equal and opposite phases. Furthermore, either their amplitude or phase must be 'locked' in the sense that it does not change with $E_{J}$. The existence of such phase- and amplitude-locked dynamics follows generically from the factorizing form of $\mathcal{H}$ found for a nonlinear drive onresonance. Indeed it also occurs in a similar manner for higher-photon resonances. The behavior of the fixed points as $E_{J}$ is increased from zero is shown in Fig. $3(\mathrm{a}, \mathrm{b})$. Initially the system behaves like a linearly driven harmonic oscillator: there is a single stable fixed point whose amplitude increases with $E_{J}$, but the phase remains locked to zero. Then $\Gamma_{+}$vanishes at the critical point $E_{J}=E_{J}^{B}$ where two stable fixed points emerge. They have the same amplitude, which locks to the fixed value $A_{0}=z_{1} / 2 \Delta_{0}$, but opposite phases.

The simplest approximation for the average current is $\left\langle I_{C P}\right\rangle=\mathcal{I}\left(\alpha, \alpha^{*}\right)$ where $\mathcal{I}$ is the classical current function, obtained by setting $a^{(\dagger)} \rightarrow \alpha^{(*)}$ in Eq. (3), with the complex amplitudes taking their fixed point values, cf. Eq. (A2). Since $\left\{A^{2}, \phi\right\}$ form a conjugate pair and $I_{C P} \sim\left[n, H_{\mathrm{RWA}}\right]$, the properties of both the resulting current and current fluctuations $\delta I$ follow from the local properties of $\mathcal{H}(A, \phi)$. The charge current $\mathcal{I}=(2 e / \hbar) \partial_{\phi} \mathcal{H}$ takes the same value for both of the fixed points when $E_{J}>E_{J}^{B}$. Comparison with numerical solu- 
tions of the full master equation in Fig. 1(a) shows that this approximation works well for $\Delta_{0} \ll 1$, though there are deviations close to the critical point and these grow progressively as $\Delta_{0}$ is increased.

The form of $\mathcal{I}\left(\alpha, \alpha^{*}\right)$ along the locus of fixed points also plays an important role for the behavior of current fluctuations. To lowest order we can approximate $\delta I=\partial_{\alpha} \mathcal{I} a+\partial_{\alpha^{*}} \mathcal{I} a^{\dagger}$, where the derivatives are evaluated at the fixed point. Figure 3(c) shows that the fixed points coincide with lines of saddle points in $\mathcal{I}$, thus $\partial_{\phi} \mathcal{I}=0$ below $\left(E_{J}<E_{J}^{B}\right)$ and $\partial_{A} \mathcal{I}=0$ above $\left(E_{J}>E_{J}^{B}\right)$ the critical point. Because of this, $\delta I=\left(\partial_{A} \mathcal{I}\right) q$ below the critical point, but $\delta I \sim\left(\partial_{\phi} \mathcal{I}\right) p / A$ as the critical point is approached from above. The maximum in $\mathcal{I}$ coincides with the critical point which suggests that the current fluctuations should vanish there. However, the very different roles that the quadratures $q$ and $p$ play near the critical point is crucial: It is in the $p$-direction, in which the fixed point splits [see Figs. 3(c) and 1(c),(d)]. Thus, $p$ is the soft mode whose dynamics becomes slow and whose fluctuations, $\left\langle p^{2}\right\rangle$, diverge at the critical point. Explicitly, we find that below the critical point, $E_{J} \leq E_{J}^{B}$, the dynamics is overdamped (real $\Gamma_{ \pm}$) with $\langle\dot{q}\rangle=-\Gamma_{-}\langle q\rangle$ and $\langle\dot{p}\rangle=-\Gamma_{+}\langle p\rangle$. Consequently, the current fluctuations below the critical point are decoupled from the critical fluctuations, while the opposite is true above.

To examine the critical scaling in detail (see also Appendix B), we quantify the distance from the critical point by $\epsilon=1-E_{J}^{B} / E_{J}$. Below the critical point, $S_{C P}=2\left\langle\delta I^{2}\right\rangle / \Gamma_{-}=2\left(\partial_{A} \mathcal{I}\right)^{2}\left\langle q^{2}\right\rangle / \Gamma_{-}$. This vanishes as the critical point is approached because $\partial_{A} \mathcal{I} \sim(A-$ $\left.A_{0}\right) \sim \epsilon$ [cf. Fig. 3(a), (c)] whilst $\Gamma_{-}$and $\left\langle q^{2}\right\rangle$ remain finite. However, when the critical point is approached from above there is a divergence: $S_{C P} \simeq 2\left\langle\delta I^{2}\right\rangle / \Gamma_{+} \simeq$ $2\left(\partial_{\phi} \mathcal{I}\right)^{2}\left\langle p^{2}\right\rangle /\left(A^{2} \Gamma_{+}\right) \sim 1 / \epsilon$ because the current fluctuations are now dominated by the critical dynamics of the soft mode: $\partial_{\phi} \mathcal{I} \sim \phi \sim \sqrt{\epsilon}$ [cf. Fig. 3(b), (c)], but $\left\langle p^{2}\right\rangle / \Gamma_{+} \sim 1 / \epsilon^{2}$.

Thus the semiclassical description predicts a discontinuity in the current noise at the critical point which in turn gives rise, smoothed over by higher order corrections, to the sudden switching in $F_{C P}$ around $E_{J}^{B}$ for small $\Delta_{0}$ (Fig. 2). Physically, this unusual behavior is a direct consequence of the cavity drive (JJ) being strongly nonlinear: the back-action of the photon field onto the photon-generating charge flow leads to a saturation of the mean current when the threshold is passed, which is accompanied by a jump in the current noise from being strongly sub-Poissonian to strongly super-Poissonian.

\section{PHOTONIC PERSPECTIVE}

In contrast to the micromaser, where measurements focused on the internal states of the atoms [46], in the JJ-cavity hybrid the behavior of the charge current is mirrored by the flow of photons leaking out of the cavity into the transmission line. The average of this photon
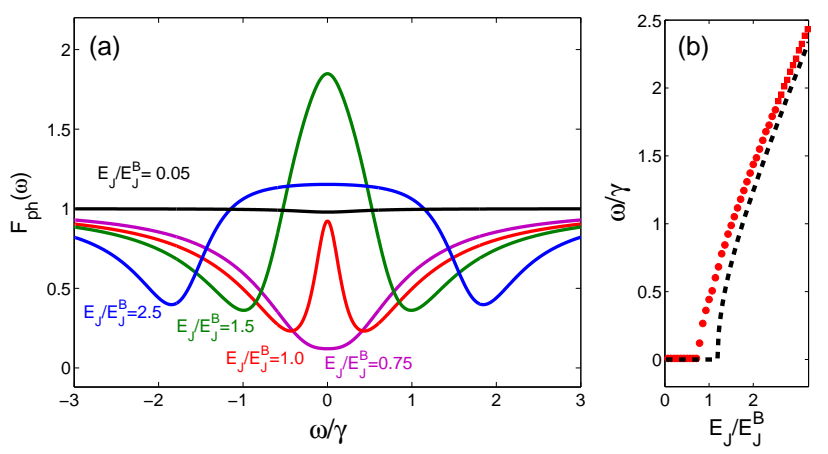

FIG. 4. (a) Evolution of the photonic Fano factor, $F_{p h}(\omega)$, with $E_{J} / E_{J}^{B}$. Calculated numerically using the full master equation with $\Delta_{0}=0.2, \delta=0$. (b) Comparison of the locations of the minima in $F_{p h}(\omega)$ (red points) with the imaginary parts of the semiclassical eigenvalues $\Gamma_{ \pm}$(black dashes).

current is $\left\langle I_{p h}\right\rangle=\gamma\langle n\rangle$ which matches $\left\langle I_{C P}\right\rangle / 2 e$. The photon current noise is $[23,47]$

$$
\begin{aligned}
S_{p h}(\omega) & =2 \operatorname{Re} \int_{0}^{\infty} d \tau \mathrm{e}^{\mathrm{i} \omega \tau}\left[\left\langle I_{p h}(t+\tau) I_{p h}(t)\right\rangle-\left\langle I_{p h}\right\rangle^{2}\right] \\
& =\left\langle I_{p h}\right\rangle+2\left\langle I_{p h}\right\rangle^{2} \operatorname{Re} \int_{0}^{\infty} d \tau \mathrm{e}^{\mathrm{i} \omega \tau}\left[g^{(2)}(\tau)-1\right],(8)
\end{aligned}
$$

where $g^{(2)}(\tau)=\left\langle a^{\dagger}(t) a^{\dagger}(t+\tau) a(t+\tau) a(t)\right\rangle /\left\langle a^{\dagger}(t) a(t)\right\rangle^{2}$, and we again assume the stationary limit $(t \rightarrow \infty)$. The fact that the photons are always generated in one-toone correspondence with the Cooper pair current means that the linkage between photonic and charge currents extends beyond their averages and we find (see Appendix C and cf. Ref. [35]) that: $F_{p h}(0)=F_{C P}$ where the Fano factor for the photon current is defined as $F_{p h}(\omega)=$ $S_{p h}(\omega) /\left\langle I_{p h}\right\rangle$. This has important consequences as it means that the charge current noise can be inferred from the photon correlation function $g^{(2)}(\tau)$, a quantity which is now routinely measured in experiments using superconducting cavities [32, 48].

As the system is tuned through the critical point, the behavior of $F_{p h}$ at $\omega=0$ simply matches that of $F_{C P}$, but there is additional information at finite frequencies, see Fig. 4. For very small $E_{J}$ the spectrum is almost flat, but a pronounced dip develops around $\omega=0$ as $E_{J}$ is increased. Growth in $F_{p h}$ at $\omega=0$ starts just before $E_{J}^{B}$ and is associated with the development of dips in $F_{p h}(\omega)$ at a finite frequency. This reflects the fact that fluctuations about the steady state are no longer overdamped - the frequencies of the minima are approximately described by emerging imaginary parts of the semiclassical eigenvalues $\Gamma_{ \pm}$. The dips move out to progressively larger frequencies as $E_{J}$ is increased further whilst $F_{p h}(0)$ gradually returns towards unity, the level set by the shot noise in the system. Notably, despite similarities in the behavior of $F_{C P}$, the transport at large $E_{J}$ is not the same as for $E_{J} \rightarrow 0$ : strong correlations remain, but at finite frequency. 


\section{CONCLUSIONS AND OUTLOOK}

We have analysed the charge transport in a voltagebiased JJ coupled to a high-Q cavity. We predict the emergence of an unusual regime of highly coherent transport of Cooper pairs and photons due to the nonlinear drive experienced by the cavity. This regime ends at a dynamical critical point where the current noise switches abruptly from sub- to super-Poissonian.

Although our theoretical treatment involves some simplifications, we nevertheless expect the main features to be readily detectable in experiment (see Appendix D for a detailed discussion). In current experimental realizations both of the circuit parameters, $E_{J}$ and $\Delta_{0}$, can be varied: $E_{J}$ by a tunable magnetic flux $[3,13]$ to move through the critical point and $\Delta_{0}$ by circuit design from the low $\left(\Delta_{0} \ll 1[2,11]\right)$ to the high $\left(\Delta_{0} \sim 1\right.$ $[49,50])$ impedance regime to study the semiclassicalquantum crossover. The switching in the noise is most easily accessible from a time-integrated measurement of $\int d \tau\left[g^{(2)}(\tau)-1\right]$, see $(8)$, which does not rely on fast detectors.

Our work also raises a number of new questions. For example, how charge and photonic dynamics behave when the critical point is passed nonadiabatically and if links to Kibble-Zurek or Landau-Zener like mechanisms can be established $[51,52]$. Further, the realization of microwave sources with tunable quantum/classical characteristics or current sources with tailored noise properties may be attractive in device applications.

\section{ACKNOWLEDGMENTS}

The authors thank M. Blencowe, S. Dambach, F. Portier and A. Rimberg for valuable discussions. The work was supported by the International Collaboration Fund of the University of Nottingham, BK and JA were also supported by Deutsche Forschungsgemeinschaft through AN336/11-1 and SFB/TRR21.

\section{Appendix A: Semiclassical Approximations}

In this Appendix we will provide additional calculational details about the semiclassical approximation. Starting points are the classical Hamiltonian and current functions given by

$$
\begin{aligned}
\mathcal{H} & =\hbar \delta|\alpha|^{2}+i \frac{\tilde{E}_{J}}{2}\left(\alpha^{*}-\alpha\right) \frac{J_{1}\left(2 \Delta_{0}|\alpha|\right)}{|\alpha|} \\
\mathcal{I} & =\frac{e \tilde{E}_{J}}{\hbar}\left(\alpha^{*}+\alpha\right) \frac{J_{1}\left(2 \Delta_{0}|\alpha|\right)}{|\alpha|} .
\end{aligned}
$$

Hence the quantities $\partial_{\alpha \alpha} \mathcal{H}$ and $\partial_{\alpha \alpha *} \mathcal{H}$ that appear in the effective quadratic Hamiltonian $H_{2}$ [Eq. (6)] are defined as follows:

$\partial_{\alpha \alpha} \mathcal{H}=i \frac{\tilde{E}_{J} \Delta_{0}^{2}}{2}\left(J_{1}\left(2 \Delta_{0} A\right) \mathrm{e}^{-i \phi}+J_{3}\left(2 \Delta_{0} A\right) \mathrm{e}^{-i 3 \phi}\right)$
$\partial_{\alpha \alpha^{*}} \mathcal{H}=\hbar \delta-\tilde{E}_{J} \Delta_{0}^{2} J_{1}\left(2 \Delta_{0} A\right) \sin \phi$

with $A$ and $\phi$ the amplitude and phase of the classical fixed point [13].

The current noise is calculated using the linear current fluctuations

$$
\delta I=\partial_{\alpha} \mathcal{I} \delta a+\partial_{\alpha^{*}} \mathcal{I} \delta a^{\dagger}
$$

with

$$
\partial_{\alpha} \mathcal{I}=\frac{e \tilde{E}_{J} \Delta_{0}}{\hbar}\left[J_{0}\left(2 \Delta_{0} A\right)-J_{2}\left(2 \Delta_{0} A\right) \mathrm{e}^{-i 2 \phi}\right] .
$$

The correlation functions for the current fluctuations can be calculated using the regression formula [53], leading to a simple expression for the current noise

$$
S_{C P}=\gamma\left|\frac{\partial_{\alpha} \mathcal{I}\left[\frac{i}{\hbar} \partial_{\alpha \alpha^{*}} \mathcal{H}-\gamma / 2\right]-\frac{i}{\hbar}\left(\partial_{\alpha^{*}} \mathcal{I}\right) \partial_{\alpha \alpha} \mathcal{H}}{\Gamma_{+} \Gamma_{-}}\right|^{2}
$$

The denominator in this expression is written in terms of the eigenvalues that describe the evolution of the linear fluctuations, $\Gamma_{ \pm}$[see Eq. (7)]. This connection is important for understanding the behavior in the vicinity of the bifurcations which the system undergoes: one of the eigenvalues vanishes at these points, implying a divergence in the noise provided the denominator remains non-zero.

Below the critical point $\left(E_{J}<E_{J}^{B}\right)$, the Fano factor can be written as

$$
F_{C P}=\frac{\left(J_{0}(z)-J_{2}(z)\right)^{2} /\left(J_{0}(z)+J_{2}(z)\right)^{2}}{\left[1+\frac{E_{J}}{E_{J}^{B}} \frac{z_{1}}{4 J_{0}\left(z_{1}\right)}\left(J_{1}(z)+J_{3}(z)\right)\right]^{2}},
$$

where $z=2 \Delta_{0} A$. In the limit $E_{J} \rightarrow 0$, the fixed point amplitude vanishes $(A \rightarrow 0)$ and hence the usual Poissonian result is recovered, $F_{C P} \rightarrow 1$, implying incoherent Cooper pair tunneling. However, as $E_{J}$ is increased the value of $F_{C P}$ drops and the semi-classical calculation predicts that $F_{C P} \rightarrow 0$ as $E_{J} / E_{J}^{B} \rightarrow 1$ since $J_{0}(z) / J_{2}(z) \rightarrow 1$ in this limit. This result occurs despite the vanishing of the denominator at the critical point because $\partial_{\alpha} \mathcal{I}$ also vanishes in this limit.

\section{Appendix B: Critical Scaling}

The fixed points of the system (defined in terms of amplitude and phase $\alpha=A \mathrm{e}^{i \phi}$ ) obey the relations,

$$
\begin{aligned}
\hbar \gamma A & =\tilde{E}_{J} \Delta_{0} \cos \phi\left[J_{0}\left(2 \Delta_{0} A\right)+J_{2}\left(2 \Delta_{0} A\right)\right] \\
2 A \hbar \delta & =-\tilde{E}_{J} \Delta_{0} \sin \phi\left[J_{0}\left(2 \Delta_{0} A\right)-J_{2}\left(2 \Delta_{0} A\right)\right],
\end{aligned}
$$


with the critical point values $z=2 \Delta_{0} A=z_{1}$ and $\phi=0$. Close to the critical point these can be written as

$$
\begin{aligned}
\left(1+\frac{\delta z}{z_{1}}\right)(1-\epsilon) & =\cos \phi \frac{\left[J_{0}\left(z_{1}+\delta z\right)-J_{1}^{\prime}\left(z_{1}+\delta z\right)\right]}{J_{0}\left(z_{1}\right)} \\
\bar{\delta} & =-\sqrt{1-\cos ^{2} \phi} J_{1}^{\prime}\left(z_{1}+\delta z\right),
\end{aligned}
$$

where we have introduced the small quantities $\delta z=z-z_{1}$ and $\epsilon=1-E_{J}^{B} / E_{J}$ to quantify the distance from the critical point; the scaled detuning, $\bar{\delta}=\delta /\left[2 \gamma J_{0}\left(z_{1}\right)\right]$, is also assumed to be small. Note that $J_{1}^{\prime}\left(z_{1}\right)=0$. By solving (B3) for $\cos \phi$ and inserting the result into (B4) and expanding in the small parameters, one obtains

$$
(\epsilon-\mu \delta z) \delta z^{2}=\left(\frac{\bar{\delta}}{\sqrt{2} J_{1}^{\prime \prime}\left(z_{1}\right)}\right)^{2}
$$

where $\mu=1 / z_{1}-\left[J_{0}^{\prime}\left(z_{1}\right)-J_{1}^{\prime \prime}\left(z_{1}\right)\right] / J_{0}\left(z_{1}\right) \simeq 1.1$.

On-resonance $\delta=0$, hence $\delta z=\epsilon / \mu$ for $\epsilon<0$ whilst $\delta z \equiv 0$ for $\epsilon>0$. The scaling of the phase for $\epsilon>0$ $(\phi \equiv 0$ for $\epsilon<0)$ is obtained by expanding $\cos \phi \simeq 1-$ $\phi^{2} / 2$ in (B3), which gives $\phi= \pm \sqrt{2 \epsilon}$. These scalings can then be used in turn to determine those of more complex functions of the fixed point amplitude and phase.

\section{Appendix C: Relationship between current Fano factors}

The approach used to demonstrate that the (zerofrequency) current Fano factor is the same at different points in a mesoscopic conductor connected between two leads [35] can be used to show that the Fano factors for the Cooper-pair and photon currents obey the relation $F_{C P}=F_{p h}(0)$. In the following we sketch out the main steps.

The analysis is carried out most conveniently using a Liouville space representation $[35,38,47,54]$ where a Hilbert space operator $b$ becomes a vector $|b\rangle\rangle$. The inner product is defined as $\langle\langle c \mid b\rangle\rangle=\operatorname{Tr}\left[c^{\dagger} b\right]$. A superoperator, $\mathcal{J}$ which maps one operator into another operator in Hilbert space maps one Liouville space vector into another vector, for example if $c=\mathcal{J} b$ then equivalently $|c\rangle\rangle=\mathcal{J}|b\rangle\rangle$. Thus, for example, the average photonic current can be re-written using Liouville space notation as

$$
\left\langle I_{p h}\right\rangle=\left\langle\left\langle 1\left|\mathcal{I}_{p h}\right| \rho_{s}\right\rangle\right\rangle,
$$

where $\left.\left|\rho_{s}\right\rangle\right\rangle$ and $\langle\langle 1|$ are Liouville vectors corresponding to the steady state density operator and the identity operator, respectively $[35,38,54]$. The superoperator $\mathcal{I}_{p h}$ is defined by $\mathcal{I}_{p h} \rho=\gamma a \rho a^{\dagger}$.

We start by noting that the zero frequency photon current noise [Eq. (8)] with $\omega=0$ ] can be written in the form $[23,35,47,54]$

$$
S_{p h}(0)=\left\langle\left\langle 1\left|\mathcal{I}_{p h}\right| \rho_{s}\right\rangle\right\rangle-2\left\langle\left\langle 1\left|\mathcal{I}_{p h} \mathcal{R} \mathcal{I}_{p h}\right| \rho_{s}\right\rangle\right\rangle
$$

where $\mathcal{R}=\mathcal{Q} \mathcal{L}^{-1} \mathcal{Q}$ is the pseudo-inverse of the Liouvillian $\mathcal{L}$ of the master equation, $\dot{\rho}=\mathcal{L} \rho$, with $\mathcal{Q}=$ $\left.1-\left|\rho_{s}\right\rangle\right\rangle\langle\langle 1|$.

To treat the Cooper-pair current and the photon current on the same footing we introduce symmetrized superoperator forms for the dc current operator

$$
\mathcal{I}_{C P} \rho=\frac{1}{2}\left(I_{C P} \rho+\rho I_{C P}\right)
$$

and the number operator, $n=a^{\dagger} a$,

$$
\mathcal{N} \rho=\frac{1}{2}(n \rho+\rho n) .
$$

Using these definitions we find

$$
[\mathcal{N}, \mathcal{L}]=\frac{1}{2 e} \mathcal{I}_{C P}-\mathcal{I}_{p h}
$$

Using this equation and the relations $\left\langle\left\langle 1|\mathcal{L}=\mathcal{L}| \rho_{s}\right\rangle\right\rangle=0$, we recover current conservation

$$
\frac{1}{2 e}\left\langle\left\langle 1\left|\mathcal{I}_{C P}\right| \rho_{s}\right\rangle\right\rangle=\left\langle\left\langle 1\left|\mathcal{I}_{p h}\right| \rho_{s}\right\rangle\right\rangle \text {. }
$$

The Cooper-pair current noise [Eq. (4)] can be written as $[35,38,54]$

$$
S_{C P}=-2\left\langle\left\langle 1\left|\mathcal{I}_{C P} \mathcal{R} \mathcal{I}_{C P}\right| \rho_{s}\right\rangle\right\rangle
$$

Although this expression for the Cooper-pair noise seems at first to be quite different to the photon noise in Eq. (C2), we can readily show that the two are in fact proportional using Eq. (C5).

Since $\left\langle\left\langle 1|\mathcal{L}=\mathcal{L}| \rho_{s}\right\rangle\right\rangle=0$ we also have $\mathcal{L} \mathcal{Q}=\mathcal{Q} \mathcal{L}=\mathcal{L}$ and hence $\mathcal{L} \mathcal{R}=\mathcal{R} \mathcal{L}=\mathcal{Q}$. Applying these relations and Eq. (C5) we obtain

$$
\frac{1}{2 e} S_{C P}=-\left\langle\left\langle 1\left|\left(\mathcal{I}_{C P} \mathcal{R} \mathcal{I}_{p h}+\mathcal{I}_{p h} \mathcal{R} \mathcal{I}_{C P}\right)\right| \rho_{s}\right\rangle\right\rangle
$$

where we have also used the fact that $\left\langle\left\langle 1\left|\left[\mathcal{I}_{C P}, \mathcal{N}\right]\right| \rho_{s}\right\rangle\right\rangle=$ 0 . Again making the substitution $(1 / 2 e) \mathcal{I}_{C P}=\mathcal{I}_{p h}+$ $[\mathcal{N}, \mathcal{L}]$ we find

$$
\begin{aligned}
\frac{S_{C P}}{4 e^{2}} & =-2\left\langle\left\langle 1\left|\mathcal{I}_{p h} \mathcal{R} \mathcal{I}_{p h}\right| \rho_{s}\right\rangle\right\rangle+\left\langle\left\langle 1\left|\left[\mathcal{I}_{p h}, \mathcal{N}\right]\right| \rho_{s}\right\rangle\right\rangle \\
& =-2\left\langle\left\langle 1\left|\mathcal{I}_{p h} \mathcal{R} \mathcal{I}_{p h}\right| \rho_{s}\right\rangle\right\rangle+\left\langle\left\langle 1\left|\mathcal{I}_{p h}\right| \rho_{s}\right\rangle\right\rangle \\
& =S_{p h}(0) .
\end{aligned}
$$

Combining this with the current conservation relation [Eq. (C6)] and the definitions, $F_{C P}=S_{C P} /\left(2 e\left\langle I_{C P}\right\rangle\right)$ and $F_{p h}(0)=S_{p h}(0) /\left\langle I_{p h}\right\rangle$, we finally obtain $F_{C P}=$ $F_{p h}(0)$.

\section{Appendix D: Experimental realization}

In the main text of the paper, we describe how to arrive at the rotating-wave Hamiltonian, $H_{\mathrm{RWA}}$, and the Lindblad-type master equation, which governs the dynamics investigated thereafter. Here, we will briefly discuss the validity of the involved approximations for experimental Josephson-cavity setups. 


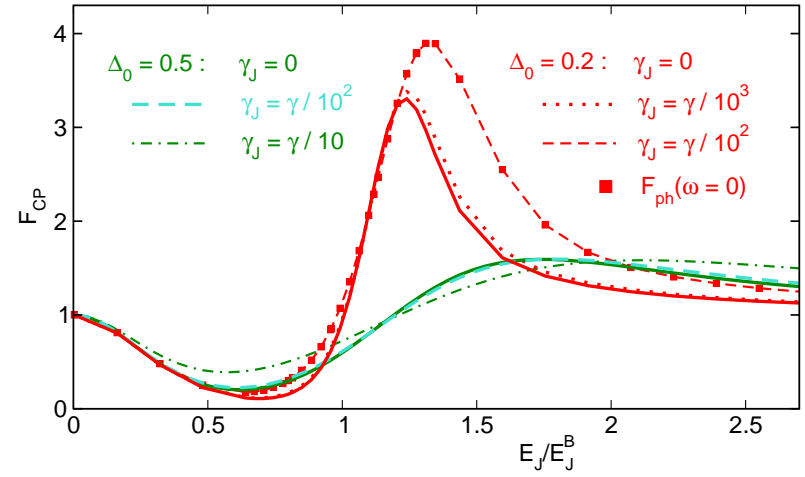

FIG. 5. Current Fano factor, $F_{C P}$, as a function of $E_{J} / E_{J}^{B}$ for large quantum fluctuations, $\Delta_{0}=0.5$ (green), and closer to the semiclassical limit, $\Delta_{0}=0.2$ (red). Results from the extended quantum-master equation incorporating an explicit junction degree of freedom converge to the approach without fluctuations of the bias voltage (solid lines, cf. Fig. 2) for $\gamma_{J} / \gamma \rightarrow 0$. The impact of low-frequency voltage noise is seen most strongly, when the system is close to the (classical) dynamical critical point, i.e., $E_{J} \approx E_{J}^{B}, \Delta_{0} \ll 1$. Notably, the exact correspondence of the current Fano factor, $F_{C P}$, and the photonic Fano factor, $F_{p h}(\omega=0)$ (symbols) accessed via $g^{(2)}$ [cf. Eq. (8)], persists for finite $\gamma_{J}$.

\section{Rotating-wave approximation}

The fact, that the Josephson coupling at the critical point, $E_{J}^{B} \sim \hbar \gamma e^{\Delta_{0}^{2} / 2} / \Delta_{0}^{2}$, can become quite large (e.g., when approaching the semiclassical regime), necessitates a closer look at the rotating wave approximation. Counter-rotating terms not included in the discussion based on Eq. (1) scale as $E_{J} / \hbar \omega_{0}$, so that validity of the RWA relies on

$$
\frac{E_{J}^{B}}{\hbar \omega_{0}} \sim \frac{\gamma}{\omega_{0} \Delta_{0}^{2}} \ll 1 \quad \Leftrightarrow \quad Q \gg \Delta_{0}^{-2} .
$$

Even for the cavities with a comparatively low quality factor, $Q \approx 40$, as employed in the most recent experiment of the Saclay group [32] non-RWA corrections would consequently be small in the relevant regime and will remain so deep into the semiclassical regime for realistically achievable $Q$ values.

\section{Voltage noise}

One important requirement for an actual experimental realization of the investigated dynamics is a sufficiently stable voltage bias across the Josephson junction. In fact, low-frequency fluctuations of the voltage constitute a further source of dissipation, which appears in addition to the damping of the cavity due to photon decay. The existence and impact of these local voltage fluctuations at the JJ are most directly apparent in the broadening of the spectrum of emitted microwave radiation $[11,14]$.

The simplest theoretical description (see the Supplementary Material of Ref. [14]) relies on explicitly including an extra degree of freedom for the number of Cooper pairs $N$ transported across the junction together with the associated junction phase $\eta$. In the RWA-Hamiltonian the $\left[a^{\dagger}-a\right]$ term is replaced by $\left[e^{i \eta} a^{\dagger}-e^{-i \eta} a\right]$, where $e^{ \pm i \eta}=\sum_{N}|N\rangle\langle N \pm 1|$. Furthermore, an additional dissipator of form $\mathcal{L}[N, \rho]=\gamma_{J}\left(2 N \rho N-N^{2} \rho-\rho N^{2}\right) / 2$ has to be appended to the master equation. Reference [14] expresses $\gamma_{J}$ in terms of circuit quantities and describes how the corresponding quantum master equation can be treated in the extended JJ-resonator space.

Extracting $\gamma_{J}$ from the experimentally observed spectral broadening one typically finds $\gamma_{J} \ll \gamma$ (see for example Ref. [11] with a ratio $\left.\gamma_{J} / \gamma \approx 0.04\right)$. Then, for many observables, e.g., for the mean cavity occupation, the oscillator damping will play the dominant role, while local voltage fluctuations can be neglected; the Hamiltonian and Liouvillian without the additional degree of freedom should thus be sufficient.

In Figure 5, we show the Fano factor resulting from such an extended description including an extra junction degree of freedom with fluctuations of varying strength, $\gamma_{J} / \gamma<1$. While voltage fluctuations have to be strongly suppressed to be completely negligible, they do not change the qualitative behavior of noise switching. As might be intuited, the low-frequency voltage fluctuations affect the Fano factor most strongly around the dynamical critical point, where the fluctuation dynamics is critically slowed down. This is in agreement with recent results in [31]. Conclusions for experiments drawn from the results shown in Fig. 5 are then the following: Lowfrequency voltage noise will not pose much of a problem for moderate quantum fluctuations. This presumably is the most immediately accessible experimental regime, since the critical Josephson energy remains small. Investigating details of the scaling in the semiclassical limit is more challenging requiring high quality cavities and excellent bias stability.
[1] O. Astafiev, K. Inomata, A. O. Niskanen, T. Yamamoto, Yu. A. Pashkin, Y. Nakamura and J. S. Tsai, Nature (London) 449, 588 (2007).

[2] F. Chen, J. Li, A. D. Armour, E. Brahimi, J. Stetten- heim, A. J. Sirois, R. W. Simmonds, M. P. Blencowe and A. J. Rimberg, Phys. Rev. B 90, 020506 (2014).

[3] M. C. Cassidy, A. Bruno, S. Rubbert, M. Irfan, J. Kammhuber, R. N. Schouten, A. R. Akhmerov, and L. 
P. Kouwenhoven, Science 355, 939 (2017).

[4] Y.-Y. Liu, J. Stehlik, C. Eichler, M. J. Gullans, J. M. Taylor and J. R. Petta, Science 347, 285 (2015).

[5] A. Stockklauser, P. Scarlino, J. V. Koski, S. Gasparinetti, C. K. Andersen, C. Reichl, W. Wegscheider, T. Ihn, K. Ensslin, and A. Wallraff Phys. Rev. X 7, 011030 (2017).

[6] H. Walther, B. T. H. Varcoe, B.-G. Englert and T. Becker, Rep. Prog. Phys. 69, 1325 (2006).

[7] P. Filipowicz, J. Javanainen, and P. Meystre, Phys. Rev. A 34, 3077 (1986).

[8] A. L. Grimsmo, F. Qassemi, B. Reulet, and A. Blais, Phys. Rev. Lett. 116, 043602 (2016).

[9] T. Holst, D. Esteve, C. Urbina, and M. H. Devoret, Phys. Rev. Lett. 73, 3455 (1994).

[10] G.-L. Ingold and Y. V. Nazarov, in Single Charge Tunneling, edited by H. Grabert and M. H. Devoret (Plenum, New York, 1992).

[11] M. Hofheinz, F. Portier, Q. Baudouin, P. Joyez, D. Vion, P. Bertet, P. Roche, and D. Esteve, Phys. Rev. Lett. 106, 217005 (2011).

[12] Y. A. Pashkin, H. Im, J. Leppäkangas, T. F. Li, O. Astafiev, A. A. Abdumalikov, E. Thuneberg, and J. S. Tsai, Phys. Rev. B 83, 020502 (2011).

[13] A. D. Armour, M. P. Blencowe, E. Brahimi and A. J. Rimberg, Phys. Rev. Lett. 111, 247001 (2013).

[14] V. Gramich, B. Kubala, S. Rohrer and J. Ankerhold, Phys. Rev. Lett. 111, 247002 (2013).

[15] S. H. Simon and N. R. Cooper, arXiv:1708.02435 (2017).

[16] M. I. Dykman and M.A. Krivoglaz, Soviet Physics JETP 50, 30 (1979).

[17] P. D. Drummond and D. F. Walls, J. Phys. A 13, 725 (1980).

[18] M. I. Dykman, in Fluctuating Nonlinear Oscillators M. Dykman (ed.), (Oxford University Press, Oxford, UK, 2012)

[19] V. Peano and M. I. Dykman, New J. Phys. 16, 015011 (2014).

[20] F. R. Ong, M. Boissonneault, F. Mallet, A. PalaciosLaloy, A. Dewes, A. C. Doherty, A. Blais, P. Bertet, D. Vion and D. Esteve, Phys. Rev. Lett. 106, 167002 (2011); F. R. Ong, M. Boissonneault, F. Mallet, A. C. Doherty, A. Blais, D. Vion, D. Esteve and P. Bertet, Phys. Rev. Lett. 110, 047001 (2013).

[21] H. J. Carmichael, Phys. Rev. X 5, 031028 (2015).

[22] W. Casteels, R. Fazio and C. Ciuti, Phys. Rev. A 95, 012128 (2017)

[23] C. Xu and M. G. Vavilov, Phys. Rev. B 88, 195307 (2013).

[24] C. Padurariu, F. Hassler and Yu. V. Nazarov, Phys. Rev. B 86, 054514 (2012).

[25] J. Leppäkangas, G. Johansson, M. Marthaler and M. Fogelström, Phys. Rev. Lett. 110, 267004 (2013).

[26] B. Kubala, V. Gramich and J. Ankerhold, Phys. Scr. T165, 014029 (2015).

[27] J. Leppäkangas, M. Fogelström, A. Grimm, M. Hofheinz, M. Marthaler and G. Johansson, Phys. Rev. Lett. 115 027004 (2015).

[28] A. D. Armour, B. Kubala and J. Ankerhold, Phys. Rev. B 91, 184508 (2015).

[29] M. Trif and P. Simon, Phys. Rev. B 92, 014503 (2015).

[30] J. Leppäkangas, M. Fogelström, M. Marthaler and G. Johansson, Phys. Rev. B 93, 014506 (2016).
[31] H. Wang, M. P. Blencowe, A. D. Armour, and A. J. Rimberg, Phys. Rev. B 96, 104503 (2017).

[32] M. Westig, B. Kubala, O. Parlavecchio, Y. Mukharsky, C. Altimiras, P Joyez, D. Vion, P. Roche, D Esteve, M. Hofheinz, M Trif, P. Simon, J. Ankerhold and F. Portier, Phys. Rev. Lett. 119, 137001 (2017).

[33] H. Grabert and G.-L. Ingold, Europhys. Lett. 58429 (2002).

[34] T. Novotný, A. Donarini and A.-P. Jauho, Phys. Rev. Lett. 90, 256801 (2003).

[35] C. Flindt, T. Novotný and A.-P. Jauho, Phys. Rev. B 70, 205334 (2004)

[36] S. D. Bennett and A. A. Clerk, Phys. Rev. B 74, 201301 (2006).

[37] O. Usmani, Ya. M. Blanter and Yu. V. Nazarov, Phys. Rev. B 75, 195312 (2007).

[38] T. J. Harvey, D. A. Rodrigues and A. D. Armour, Phys. Rev. B 78, 024513 (2008).

[39] V. Koerting, T. L. Schmidt, C. B. Doiron, B. Trauzettel, and C. Bruder, Phys. Rev. B 79, 134511 (2009).

[40] J. Brüggemann, G. Weick, F. Pistolesi, and F. von Oppen, Phys. Rev. B 85, 125441 (2012).

[41] N. Lambert, F. Nori and C. Flindt Phys. Rev. Lett. 115, 216803 (2015)

[42] This treatment can also be generalised to include the effects of low frequency voltage fluctuations, see Appendix D 2 and $[14,31]$.

[43] J. R. Johansson, P. D. Nation and F. Nori, Comp. Phys. Comm. 183, 1760 (2012); J. R. Johansson, P. D. Nation, and F. Nori, Comp. Phys. Comm. 184, 1234 (2013).

[44] Y. M. Blanter and M. Büttiker, Phys. Rep. 336, 1 (2000).

[45] Written in terms of amplitude and phase, the fixed point relation $\partial_{\alpha} \mathcal{H}=-i \hbar \gamma \alpha^{*} / 2$ gives rise to the pair of equations, $\partial_{A} \mathcal{H}=0$ and $\partial_{\phi} \mathcal{H}=\hbar \gamma A^{2}$, only one of which depends on $\gamma$ explicitly.

[46] G. Rempe and H. Walther, Phys. Rev. A 42, 1650 (1990).

[47] C. Emary, C Pöltl, A. Carmele, J. Kabuss, A. Knorr and T. Brandes, Phys. Rev. B 85, 165417 (2012).

[48] D. Bozyigit, C. Lang, L. Steffen, J. M. Fink, C. Eichler, M. Baur, R. Bianchetti, P. J. Leek, S. Filipp, M. P. da Silva, A. Blais, and A. Wallraff, Nat. Phys. 7, 154 (2011); C. Lang, D. Bozyigit, C. Eichler, L. Steffen, J. M. Fink, A. A. Abdumalikov, Jr., M. Baur, S. Filipp, M. P. da Silva, A. Blais, and A. Wallraff Phys. Rev. Lett. 106, 243601 (2011); C. Lang, C. Eichler, L. Steffen, J. M. Fink, M. J. Woolley, A. Blais, and A. Wallraff, Nat. Phys. 9, 345 (2013).

[49] M. A. Castellanos-Beltran and K. W. Lehnert, Appl. Phys. Lett. 91, 083509 (2007).

[50] C. Altimiras, O. Parlavecchio, P. Joyez, D. Vion, P. Roche, D. Esteve and F. Portier, Phys. Rev. Lett. 112, 236803 (2014).

[51] W. Casteels, F. Storme, A. Le Boité and C. Ciuti, Phys. Rev. A 93, 033824 (2016).

[52] L. Henriet and K. Le Hur, Phys. Rev. B 93, 064411 (2016).

[53] H. J. Carmichael, Statistical Methods in Quantum Optics, Vol. 1 (Springer, Heidelberg, 1999).

[54] C. Flindt, T. Novotný and A.-P. Jauho, Europhys. Lett. 69, 475 (2005). 